Note

\section{Effect of Pressure on the Sol-Gel Transition of Agarose}

\author{
Kunihiko Gekko and Mika Fukamtzu \\ Department of Food Science and Technology, \\ Faculty of Agriculture, \\ Nagoya University, \\ Aichi 464, Japan
}

Received March 13, 1991

The mechanism for the gelation of food macromolecules has been extensively studied by various types of experiments. However, there are only limited data for pressure effects on the sol-gel transition of thermoreversible gels. ${ }^{1-3)}$ Recently, we have found that gelatin gel was stabilized under high pressure, ${ }^{3)}$ while carrageenan gel was conversely destabilized, ${ }^{2)}$ despite the cross-linking junctions of both gels being commonly formed by the hydrogen-bonding mechanism. These findings indicate that a high pressure study could be a diagnostic test not only for the volume change but also for the mechanism of gelation. In this paper, we report the pressure effect on the sol-gel transition of agarose in comparison with that of carrageenan and gelatin.

A commercial grade of agarose from Sigma Chemical Co. (Type IX, sulfate content $<0.10 \%$ ) was dissolved in distilled water at $45^{\circ} \mathrm{C}$ and gelled at $0^{\circ} \mathrm{C}$ for $48 \mathrm{hr}$ in the small glass test-tube containing a small stainless steel ball. The test-tube was sealed with Parafilm and set bottom up in the light path of a high-pressure vessel with sapphire windows, which was made to order by Kobe Steel Ltd. After the system had been equilibrated at the working pressure, the temperature of the sample was increased at a rate of $0.1^{\circ} \mathrm{C} / \mathrm{min}$, and the temperature at which the transmitted light at $400 \mathrm{~nm}$ was intercepted by the bail dropping is defined as the melting temperature of the gel, $T_{\mathrm{m}}$, at the defined pressure. The detailed experimental procedures are described in the previous papers. ${ }^{2,3)}$

Figure 1 shows the pressure dependence of $T_{\mathrm{m}}$ at various agarose concentrations: the phases above and below each line correspond to the sol and gel states, respectively. Evidently, $T_{\mathrm{m}}$ increased linearly with increasing pressure, indicating that agarose gel was stabilized under high pressure. In all cases, the slope, $\mathrm{d} T_{\mathrm{m}} / \mathrm{d} P$, appeared to be independent of the agarose concentration. The $\mathrm{d} T_{\mathrm{m}} / \mathrm{d} P$ value averaged for the agarose concentrations examined is listed in the second column of Table I. As shown in the inset to Fig. 1, the logarithmic agarose concentration, $c$, is a linear function of $1 / T_{\mathrm{m}}$ (Eldridge-Ferry plots) ${ }^{4 /}$ at any pressure. This allows us to estimate the heat of gel formation, $\Delta H$, from the slope by using the equation ${ }^{4}$ $\log c=\Delta H / 2.303 \mathrm{RT}_{\mathrm{m}}+$ const. The corresponding entropy change, $\Delta S$, and volume change, $\Delta V$, were calculated at $25^{\circ} \mathrm{C}$ from the Clausius-Clapeyron equation, $\mathrm{d} T_{\mathrm{m}} / \mathrm{d} P=$ $T \triangle V / \triangle H=\Delta V / \triangle S$, assuming that the sol-gel transition was a phase transition. The results of the calculation are listed in Table I. It should be noted that the thermodynamic parameters thus obtained are apparent and expressed as

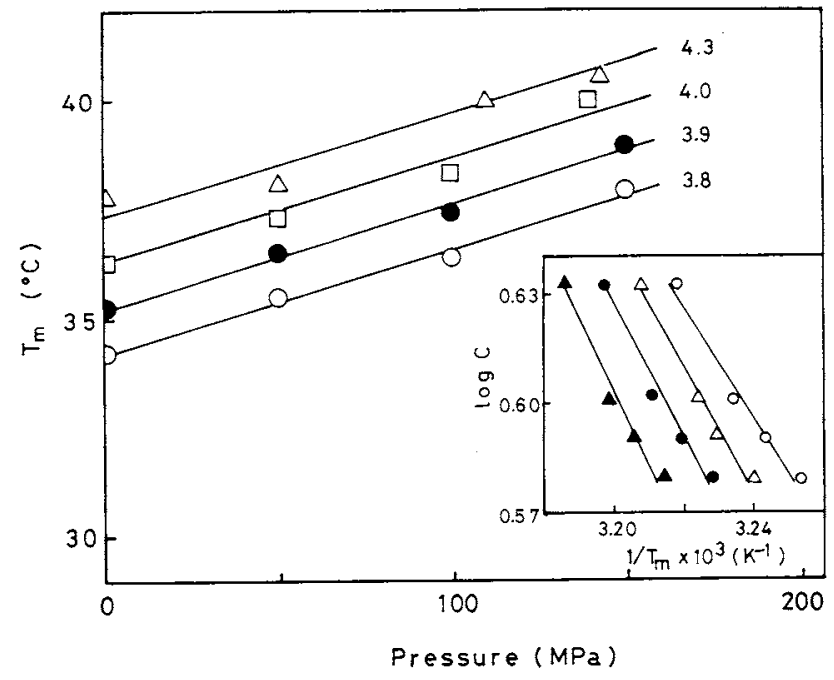

Fig. 1. Effect of Pressure on the Melting Temperature, $T_{\mathrm{m}}$, of Agarose Gel.

The numbers attached to each line represent the agarose concentration, $c$, in $\mathrm{g} / \mathrm{l}$. The inset to the figure represents plots of the logarithmic agarose concentration as a function of the reciprocal of the melting temperature of the gels at various pressures: $O, 1 \mathrm{MPa}, \triangle, 50 \mathrm{MPa} ; 100 \mathrm{MPa} ; \boldsymbol{\Delta}, 150 \mathrm{MPa}$. 
Table I. Thermodynamic Parameters of The Sol $\rightarrow$ Gel Transition of Agarose in Water at $25^{\circ} \mathrm{C}$

\begin{tabular}{rcccc}
\hline $\begin{array}{c}\text { Pressure } \\
(\mathrm{MPa})\end{array}$ & $\begin{array}{c}\mathrm{d} T_{\mathrm{m}} / \mathrm{d} P \\
(\mathrm{~K} / \mathrm{MPa})\end{array}$ & $\begin{array}{c}\Delta H \\
(\mathrm{~kJ} / \mathrm{mol})\end{array}$ & $\begin{array}{c}\Delta S \\
(\mathrm{~J} / \mathrm{K} \cdot \mathrm{mol})\end{array}$ & $\begin{array}{c}\Delta V \\
(\mathrm{ml} / \mathrm{mol})\end{array}$ \\
\hline 1 & & -34.2 & -115 & -2.6 \\
50 & $2.3 \times 10^{-2}$ & -36.4 & -122 & -2.8 \\
100 & & -36.9 & -124 & -2.9 \\
150 & & -39.5 & -132 & -3.1 \\
\hline
\end{tabular}

$\Delta H, \Delta S$ and $\Delta V$ are expressed in units per mol of cross-linked junctions.

the quantities per mol of cross-links which may correspond to the intermolecular hydrogen-bonding junctions. The values of $\Delta H$ and $\Delta S$ are negative, as was found for gelatin and carrageenan, indicating that the stabilization of agarose gel was predominantly brought about by a large exothermic effect overcoming the loss of conformational entropy due to the intermolecular hydrogen bonds. However, the sign of $\Delta V$ is negative for agarose, in contrast to that for carrageenan, although a similar gelation mechanism (intermolecular double-helix junction) has been proposed for both polysaccharides. ${ }^{5)}$ Generally, the intermolecular association of polymers produces a positive volume change in water, since the water of hydration contracted around the polymer chains is released by that process. $^{6,7)}$ The negative $\Delta V$ value for agarose cannot, therefore, be explained by such a simple hydration model, while it is applicable to carrageenan gel. ${ }^{2)}$ Although there are some arguments on the hydration states of agarose gel, ${ }^{87}$ the negative volume change might be possible if the cross-linking junctions consist of intermolecular hydrogen bonds mediated through the bridging water molecules, as has been demonstrated for gelatin gel which also shows negative $\Delta V$ values. ${ }^{3)}$ The small absolute values of $\Delta V$ suggest that the size of the cross-links in agarose gel is considerably smaller than those of carrageenan and gelatin gels. Although the origin of the volume change should be discussed in more detail on a molecular level, the present study could lead to a new characterization of the cross-links of agarose gel.

Acknowledgments. This study was supported by a Grant-in-Aid for Scientific Research (No. 62560123) from the Ministry of Education, Science and Culture of Japan and by The Nakano Foundation.

\section{References}

1) K. Suzuki, Y. Taniguchi and T. Enomoto, Bull. Chem. Soc. Jpn., 45, 336 (1972).

2) K. Gekko and K. Kasuya, Int. J. Biol. Macromol., 7, 299 (1985).

3) K. Gekko and M. Fukamizu, Int. J. Biol. Macromol., in press.

4) J. E. Eldridge and J. D. Ferry, J. Phys. Chem., 58, 992 (1954).

5) V. J. Morris, in "Functional Properties of Food Macromolecules," ed. by J. R. Mitchell and P. A. Ledward, Elsevier Applied Science Publishers, London, 1986, Chapter 3.

6) K. Heremans, Ann. Rev. Biophys. Bioeng., 11, 1 (1982).

7) K. Gekko, in "Pressure-Processed Food," ed. by R. Hayashi, San-Ei Shuppan Co., Kyoto, 1990, Chapter 2.

8) A. Suggett, in "Water," Vol. 4, ed. by F. Franks, Plenum Press, New York, 1975, Chapter 6. 